\title{
Maladaptive Plasticity in Aphasia: Brain Activation Maps Underlying Verb Retrieval Errors
}

\author{
Kerstin Spielmann, ${ }^{1,2}$ Edith Durand, ${ }^{3}$ Karine Marcotte, ${ }^{4}$ and Ana Inés Ansaldo ${ }^{3,4}$ \\ ${ }^{1}$ Rijndam Rehabilitation Institute, P.O. Box 23181, 3001 KD Rotterdam, Netherlands \\ ${ }^{2}$ Erasmus MC, University Medical Center Rotterdam, Department of Rehabilitation Medicine, P.O. Box 2040, \\ 3000 CA Rotterdam, Netherlands \\ ${ }^{3}$ Centre de Recherche de l'Institut Universitaire de Gériatrie de Montréal, 4565 Chemin Queen-Mary, \\ Montréal, QC, Canada H3W 1W5 \\ ${ }^{4}$ École d'Orthophonie et d'Audiologie, Université de Montréal, 7077 Avenue du Parc, Montréal, QC, Canada H3N 1X7 \\ Correspondence should be addressed to Kerstin Spielmann; kspielmann@rijndam.nl
}

Received 16 November 2015; Revised 25 April 2016; Accepted 5 May 2016

Academic Editor: Malgorzata Kossut

Copyright @ 2016 Kerstin Spielmann et al. This is an open access article distributed under the Creative Commons Attribution License, which permits unrestricted use, distribution, and reproduction in any medium, provided the original work is properly cited.

\begin{abstract}
Anomia, or impaired word retrieval, is the most widespread symptom of aphasia, an acquired language impairment secondary to brain damage. In the last decades, functional neuroimaging techniques have enabled studying the neural basis underlying anomia and its recovery. The present study aimed to explore maladaptive plasticity in persistent verb anomia, in three male participants with chronic nonfluent aphasia. Brain activation maps associated with semantic verb paraphasia occurring within an oral picture-naming task were identified with an event-related fMRI paradigm. These maps were compared with those obtained in our previous study examining adaptive plasticity (i.e., successful verb naming) in the same participants. The results show that activation patterns related to semantic verb paraphasia and successful verb naming comprise a number of common areas, contributing to both maladaptive and adaptive neuroplasticity mechanisms. This finding suggests that the segregation of brain areas provides only a partial view of the neural basis of verb anomia and successful verb naming. Therefore, it indicates the importance of network approaches which may better capture the complexity of maladaptive and adaptive neuroplasticity mechanisms in anomia recovery.
\end{abstract}

\section{Introduction}

Anomia, or impaired word retrieval, is the most prominent and widespread symptom of aphasia, an acquired language impairment that can result from a focal brain lesion [1]. In the context of oral word retrieval, different types of errors (i.e., paraphasia) can occur, including phonemic paraphasia, semantic paraphasia, neologisms, and circumlocutions (i.e., using devious ways to describe words) [2].

The present study focuses on semantic paraphasia in the context of verb retrieval. Verbs carry a critical meaning since they have important functions in the structural formulation of sentences [3]. Therefore, verb paraphasia has a considerable impact on an individual's capacity to convey meaning, which can lead to a substantial handicap. Semantic verb paraphasia occurs when a target verb is replaced by a semantically related verb [4], such as saying "running" instead of "walking." Research on the cognitive mechanisms underlying the production of semantic paraphasia shows that these may result from impaired phonological processing or impaired semantic processing or a combination of both [5].

Functional neuroimaging techniques allow studying the neural basis underlying verb production and anomia and its recovery. The neural substrate of verb production involves a left frontal cortical network, including the left prefrontal cortex [6], the left superior parietal lobule, the left superior temporal gyrus [7], the left superior frontal gyrus [8], and the primary motor cortex, in the posterior portion of the precentral gyrus [9-11]. In the context of verb anomia, the production of semantic paraphasia may reflect damage of these language-related areas, as well as an attempt to compensate for the impairments resulting from this brain damage as 
there is a semantic relation between the target and response [12]. This attempt to compensate can be related to the concept of neuroplasticity which refers to a number of brain mechanisms involved in learning and relearning and can be reflected by changes in brain activation patterns highlighted by functional magnetic resonance imaging (fMRI).

Two main forms of neuroplasticity have been studied: functional reactivation, which occurs when previously damaged and inactive areas recover their function after a latency period [13], and functional reorganization, which reflects compensation of the permanent damage of specific brain areas by the recruitment of some other areas not previously involved in language processing [12]. Different types of neuroplasticity may occur during anomia recovery: if this results in functional recovery (as reflected by successful word retrieval), neuroplasticity is defined as adaptive, whereas when errors (such as paraphasia) persist neuroplasticity is considered to be maladaptive $[14,15]$.

There is an ongoing debate regarding the functional reorganization in anomia recovery and whether these compensatory processes reflect adaptive or maladaptive plasticity. The left cerebral hemisphere (LH) is considered the dominant hemisphere in language processing, at least in righthanded individuals [17]. The fMRI literature has many reports in which LH damage is followed by a shift of language processing to the right cerebral hemisphere (RH), that is, laterality shift $[18-21]$. However, the extent to which this RH shift reflects adaptive or maladaptive neuroplasticity remains controversial. Some studies focus on the benefits of $\mathrm{RH}$ recruitment [22] and emphasize the role of the RH in language processing in healthy subjects [23]. Others suggest that $\mathrm{RH}$ recruitment leads to persistent errors, reflecting maladaptive plasticity [24]. Compared to the LH, the RH may have broad overlapping semantic maps: in this case, lexical selection processing would be less semantically specified and would be associated with semantic paraphasia [25]. Another view is that $\mathrm{RH}$ recruitment could be beneficial in the short term whereas, in the long term, it could contribute to an incomplete or less efficient improvement compared with a better recovery sustained by the reactivation of $\mathrm{LH}$ language processing areas [19-21, 26-28]. Moreover, the extent to which $\mathrm{RH}$ recruitment is adaptive or maladaptive may depend on lesion size $[12,27]$. These latter authors argue that while minimal damage to core language processing areas leads to maladaptive $\mathrm{RH}$ recruitment, extended $\mathrm{LH}$ lesions may trigger adaptive $\mathrm{RH}$ recruitment by release of the $\mathrm{RH}$ potential to process language. Overall, the literature presents a largely negative view on the impact of $\mathrm{RH}$ recruitment in the context of aphasia and anomia recovery, in particular in cases of moderate LH damage.

One way of examining the extent of $\mathrm{LH}$ and $\mathrm{RH}$ recruitment in anomia recovery is by calculating a lateralization index (LI) using fMRI data. The LI reflects hemispheric dominance in terms of the number of activated voxels observed in the context of a specific language task [29]. This index can express the relative contribution of either hemisphere to the processing of specific information, which can be linked to behavioral performance. Several studies have examined the relative contribution of either cerebral hemisphere to anomia recovery within the context of specific and intensive language therapy and by reference to principles of experience-dependent neuroplasticity, derived from animal research $[14,15]$. These studies investigated the neurofunctional markers of adaptive plasticity and link right and left hemisphere performance to posttherapy behavior by correlating activation patterns to posttherapy scores on naming tasks [30, 31].

Other studies used noninvasive brain stimulation techniques to modulate cortical excitability in either hemisphere, using repetitive transcranial magnetic stimulation (rTMS) and transcranial direct current stimulation (tDCS). rTMS generates magnetic fields and this can either activate or inhibit neurons. rTMS inhibiting RH areas can significantly reduce speech-error production in nonfluent aphasia [32, 33]. Inhibiting the right pars triangularis (part of the right inferior frontal gyrus) with rTMS improves naming accuracy and decreases naming latency, while activating the right pars opercularis decreases naming accuracy and improves naming latency [33]. With tDCS, a low current can be applied to the brain and, depending on the polarity, it can either enhance (anodal tDCS) or inhibit neural activity (cathodal tDCS) in a certain area. Studies using tDCS mostly combine tDCS with word-finding therapy and find an additional effect of $\mathrm{tDCS}$ on naming performance [34,35]. In summary, rTMS/tDCS studies aim to modulate adaptive plasticity, either by inhibiting $\mathrm{RH}$ areas or by enhancing $\mathrm{LH}$ areas.

In general, most of the fMRI literature on the recovery from anomia adopts a segregation approach in the analysis of fMRI activation patterns. This is a within-area approach, based on activation changes occurring in isolation [36]. For example, a brain area found to be critical in successful naming is the left Brodmann area 22, which includes the superior temporal gyrus [12,37]. Another perspective, the integration perspective, gathers brain activation patterns within coherent networks supporting a specific behavior; for example, functional connectivity analysis can be used to study networks of language processing in healthy and braindamaged populations $[38,39]$.

In summary, research on the neural basis of anomia recovery has mostly focused on segregating brain areas whose activation is associated either with persistent anomia (i.e., paraphasia), reflecting maladaptive neuroplasticity, or with recovery (i.e., successful naming), reflecting adaptive neuroplasticity. Within this perspective, rTMS/tDCS has been used to modulate $\mathrm{RH}$ takeover by inhibiting $\mathrm{RH}$ areas, traditionally associated with maladaptive neuroplasticity, or by enhancing $\mathrm{LH}$ areas related to adaptive neuroplasticity. However, there is limited knowledge regarding the specific areas whose activation is associated either with the production of paraphasia or with successful naming.

The present study aims to examine maladaptive and adaptive neuroplasticity processes in the context of verb anomia recovery in aphasia. Three participants with nonfluent chronic aphasia were examined in the context of a picturenaming task during event-related fMRI scanning. Activation patterns related to the production of semantic paraphasia were obtained and compared with our previous study that 


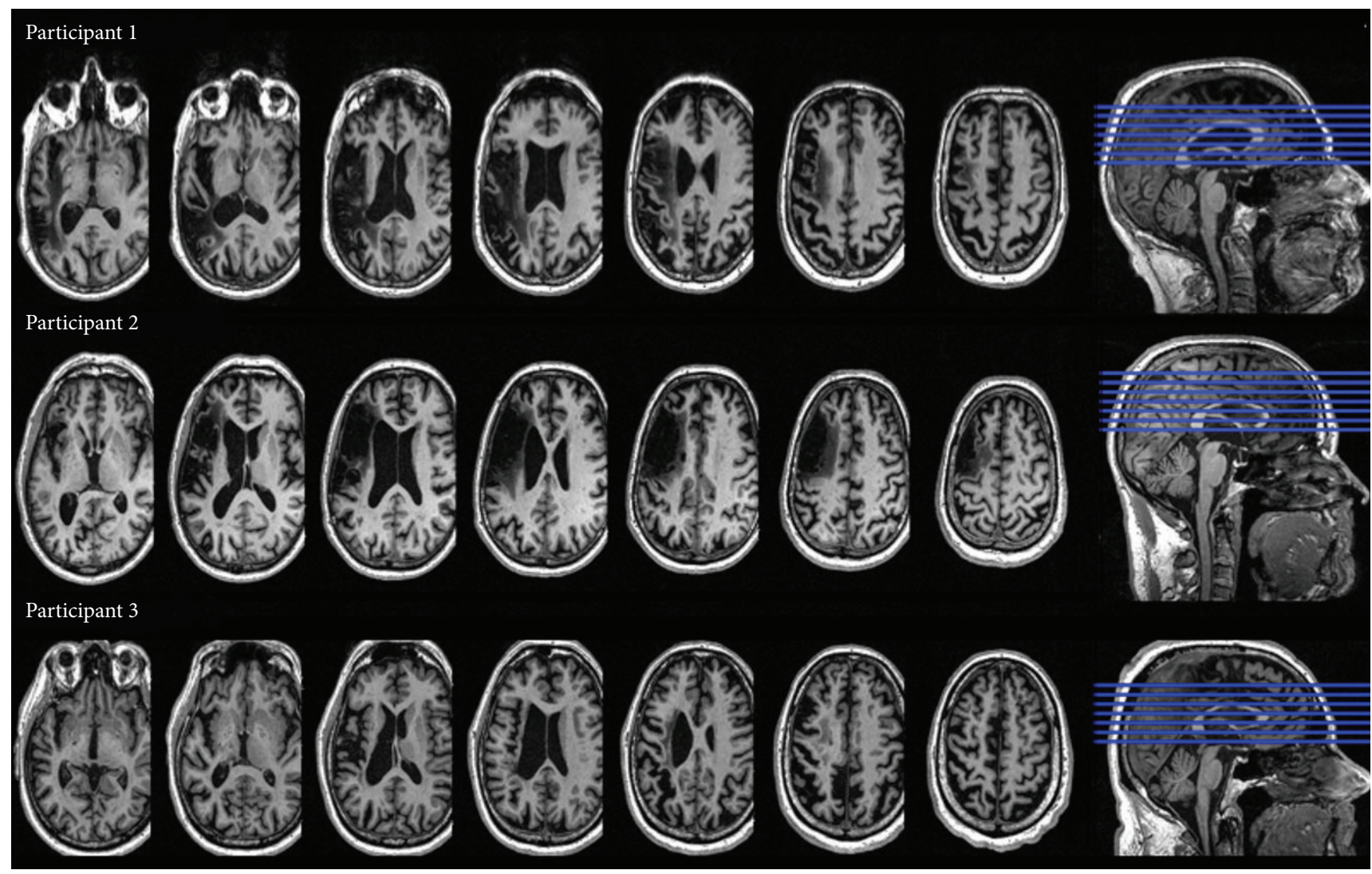

Figure 1: Lesion location for Participant 1, Participant 2, and Participant 3.

focused on adaptive plasticity, that is, successful verb naming [16]. The relative contribution of the $\mathrm{LH}$ and $\mathrm{RH}$ to semantic paraphasia and successful naming is explored by calculating an LI.

\section{Materials and Methods}

2.1. Experimental Design. The fMRI blood oxygenation leveldependent (BOLD) responses associated with the production of semantic paraphasia produced in the context of verb naming were compared to those related to successful verb naming. BOLD responses were collected in the context of an oral picture-naming verb task within an event-related fMRI paradigm.

2.2. Participants. Three male participants from the sample of Marcotte et al. [40], diagnosed with moderate to severe Broca's aphasia, were examined. Inclusion criteria were as follows: (1) a single LH stroke, (2) a diagnosis of moderate to severe aphasia, according to the Montreal-Toulouse battery [41], (3) the presence of anomia in a standardized naming task [42], (4) having French as their mother tongue, and (5) being right-handed prior to the stroke. Exclusion criteria were as follows: (1) the presence of a neurological or psychiatric diagnosis other than stroke, (2) incompatibility with fMRI testing, or (3) a diagnosis of mild cognitive impairment or dementia prior to stroke, based on medical charts, speech-pathology
TABle 1: Demographic characteristics of the three participants (adapted from Durand [16]).

\begin{tabular}{lccc}
\hline & Participant 1 & Participant 2 & Participant 3 \\
\hline Age (years) & 67 & 67 & 66 \\
Gender & Male & Male & Male \\
Months after stroke & 72 & 54 & 241 \\
Years of education & 20 & 15 & 12 \\
Lesion volume $\left(\mathrm{cm}^{3}\right)$ & 167.84 & 117.84 & 84.77 \\
\hline
\end{tabular}

reports, and information from the family. The study was approved by the Ethics Committee of the Regroupement Neuroimagerie/Québec (Canada); all participants provided written informed consent.

Lesion location differed between the participants. Participant 1 (P1) presented a left frontoparietal-temporal lesion, whereas Participants 2 (P2) and 3 (P3) presented a left frontotemporal lesion (Figure 1).

Table 1 presents demographic data; participants were comparable in terms of age and chronic status, and all had extended brain lesions in the left hemisphere (chi-square test: age, $p=0.223$; months after stroke, $p=0.199$; years of education, $p=0.199$; lesion volume, $p=0.199$ ). 


\subsection{Procedure}

2.3.1. Language Assessment. Aphasia profiles were determined with the Montreal-Toulouse 86 [41]. To ensure stable performance, two baseline naming assessments were obtained before the fMRI study. This baseline assessment was used to select stimuli for the Semantic Feature Analysis therapy, in order to provide personalized therapy (for details, see Marcotte et al. [40]). The selection was done on the basis of individual performance on the Snodgrass and Vanderwart items [42], including object images, and ColorCards ${ }^{\circledR}$ [43], including pictures depicting action verbs.

The present study focused on the ColorCards [43] which included 120 pictures. A total of 80 pictures (60 incorrectly named verbs and 20 correctly named verbs) were selected for the oral picture-naming task during the fMRI session. In addition, 20 digitally distorted images of a subset of these pictures were added as control stimuli.

2.3.2. fMRI Session: Stimuli and Procedure. Participants underwent a practice session in the mock scanner to become accustomed to the scanner noise and environment during the fMRI session. During this session, they were also trained to avoid head movements while naming the stimuli. The stimuli for the picture-naming task (ColorCards) and the control stimuli (i.e., computerized distorted pictures) were projected on a white background by means of a series of mirrors and in a random fashion. Each picture was presented for $4500 \mathrm{~ms}$ with an interstimulus interval ranging from 4500 to $8500 \mathrm{~ms}$. Participants were asked to name the pictures representing verbs as accurately as possible, avoiding head movements. In the control condition, participants had to say "BABA" when a computerized distorted picture was presented. Oral and event-related BOLD responses were collected.

2.3.3. Functional Neuroimaging Parameters. Images were acquired using a 3T MRI Siemens Trio scanner, with a standard 8-channel head coil. The image sequence was a $\mathrm{T}^{*}$-weighted pulse sequence $(\mathrm{TR}=2200 \mathrm{~ms}$; TE $=30 \mathrm{~ms}$; matrix $=64 \times 64$ voxels; $\mathrm{FOV}=192 \mathrm{~mm}$; flip angle $=90^{\circ}$; slice thickness $=3 \mathrm{~mm}$; acquisition $=36$ slides in the axial plane, with a distance factor of $25 \%$, so as to scan the whole brain, including the cerebellum). A high-resolution structural image was obtained before the two functional runs using a $3 \mathrm{D}$ T1-weighted pulse sequence $(\mathrm{TR}=2300 \mathrm{~ms}$; $\mathrm{TE}=$ $2.91 \mathrm{~ms} ; 160$ slices; matrix $=256 \times 256 \mathrm{~mm}$; voxel size $=1 \times$ $1 \times 1 \mathrm{~mm} ; \mathrm{FOV}=256 \mathrm{~mm}$ ). The protocol was designed in an event-related fashion so that BOLD responses corresponding to each image could be identified.

\subsection{Data Analysis}

2.4.1. Behavioral and fMRI Data Analysis. Average response times and error rates were calculated for four subtypes of errors: semantic paraphasia, phonological paraphasia, neologism, and circumlocutions. Only semantic paraphasia was produced in a sufficient number to perform fMRI data analysis for all three participants. Therefore, the eventrelated fMRI responses to semantic paraphasia were analyzed
TABLE 2: Error rates and the type of paraphasia produced by each participant.

\begin{tabular}{lccc}
\hline & Participant 1 & Participant 2 & Participant 3 \\
\hline Semantic paraphasia & 60 & 15 & 47 \\
Phonological paraphasia & 0 & 0 & 0 \\
Neologism & 0 & 0 & 0 \\
Circumlocution & 0 & 32 & 0 \\
\hline
\end{tabular}

following the same procedures as described by Marcotte et al. [40] and Durand [16]. Activation maps were obtained for each participant by subtracting BOLD responses in the control condition from those obtained in the trials where the answer provided was semantic paraphasia. $t$-tests, performed on each voxel, were considered significant with a cluster size $(k) \geq 10$ voxels and a $p$ value $<0.005$. Individual activation maps, including significantly activated brain areas, were determined within the framework of the Talairach atlas [44] and transformed from Talairach space to the spatial coordinates in the Montreal Neurological Institute space [45]. BOLD responses on successful verb naming were examined in our previous study that included the same three participants [16]. In this previous study, BOLD responses in the control condition were subtracted from those obtained in the trials where the answer provided was a correct answer.

Furthermore, an LI [29] was calculated for each participant to estimate the relative contribution of the $\mathrm{LH}$ and the $\mathrm{RH}$ to the production of semantic paraphasia and successful naming, respectively. Regarding successful naming, data from Durand [16] were used. We applied Lehéricy's algorithm [29], as follows: $(\mathrm{LH}-\mathrm{RH}) /(\mathrm{LH}+\mathrm{RH})$, by which a positive LI corresponds to a $\mathrm{LH}$ dominant contribution; strong left lateralization is represented by an LI ranging from 0.5 to 1.0 , and weak left lateralization is represented by an LI ranging from 0.25 to 0.5 . A negative LI corresponds to a predominant $\mathrm{RH}$ contribution; strong right lateralization is represented by an LI ranging from -1.0 to -0.5 , and weak right lateralization is represented by an LI ranging from -0.5 to -0.25 . An LI ranging from -0.25 to 0.25 represents a symmetric contribution of the left and right hemispheres to processing.

\section{Results and Discussion}

3.1. Behavioral Results. Average response times were calculated for paraphasia production; however, due to technical issues these data were not available for analysis. For the 80 pictures, Table 2 presents the error rates and the types of paraphasia produced by each participant during the event-related fMRI study. P1 produced 60 semantic paraphasias and 20 correct responses; P2 produced 15 semantic paraphasias, 32 circumlocutions, and 33 correct responses; and $\mathrm{P} 3$ produced 47 semantic paraphasias and 33 correct responses. Only semantic paraphasias were produced in a sufficient number to perform fMRI data analysis for all three participants. 


\section{2. fMRI Results}

3.2.1. Single-Subject Brain Activation Maps. Brain activation maps corresponding to maladaptive plasticity, that is, production of semantic paraphasia, in each participant are summarized in Tables 3(a)-3(c). In P1, the production of semantic paraphasia was observed concurrently with significant activation of the precentral gyrus bilaterally, the left superior frontal gyrus (SFG), the inferior frontal gyrus (IFG) bilaterally, the cerebellum (culmen bilaterally, right cerebellar tonsil), the left middle frontal gyrus (MFG), the left brain stem (pons), the left postcentral gyrus, the left fusiform gyrus, the right posterior cingulate cortex, and the right superior temporal gyrus (STG). In P2, the production of semantic paraphasia was observed concurrently with significant activation of the left thalamus (ventral lateral nucleus), the left inferior temporal gyrus (ITG), the cerebellum (left inferior semilunar lobule, right tuber), the right cuneus, the right MFG, the right IFG, the right STG, the right precuneus, the right precentral gyrus, the right middle temporal gyrus (MTG), and the right posterior cingulate cortex. Finally, in P3, the production of semantic paraphasia was observed concurrently with significant activation of the MTG bilaterally, the IFG bilaterally, the left superior parietal lobule, the left inferior parietal lobule, the right precentral gyrus, the right cingulate gyrus, the right SFG, the right putamen, the right MFG, and the right insula.

Table 4 summarizes brain activation maps corresponding to adaptive plasticity (i.e., successful naming) in each participant, adapted from Durand [16]. Successful naming was observed concurrently with significant activation of the MFG bilaterally and the precentral gyrus bilaterally. For the LH, successful naming was observed concurrently with significant activation of the IFG, the SFG, the middle occipital gyrus, the lingual gyrus, the superior parietal lobule, the precuneus, and the pons. For the RH, successful naming was observed concurrently with significant activation of the STG, the MTG, the ITG, the cerebellum (tuber and inferior semilunar lobule), the fusiform gyrus, the sulcus callosomarginalis, and the caudate nucleus.

A comparison was made between brain activation maps associated with semantic paraphasia and those associated with successful naming. In all participants, brain activation maps associated with semantic paraphasia and those associated with successful naming included a number of common significant activation patterns. These common significant activation patterns are highlighted in Tables 3(a)-3(c). In $\mathrm{P} 1$, the areas significantly activated with both semantic paraphasia and successful naming included the precentral gyrus bilaterally, the left brainstem (pons), and the right STG. In P2, the areas significantly activated with both semantic paraphasia and successful naming included the cerebellum (tuber), the right STG, and the right MTG. Finally, in P3, the areas significantly activated with both semantic paraphasia and successful naming included the left IFG and the left superior parietal lobule.

3.2.2. Lateralization Indexes. Table 5 presents the LI for the brain activation maps related to maladaptive plasticity (production of semantic paraphasia) and adaptive plasticity (successful naming) for each participant.

The three participants showed bilateral significant activation patterns for both semantic paraphasia and successful naming. Regarding the production of semantic paraphasia, two distinct patterns were observed. Whereas P1 presented a symmetric activation pattern $(-0.11), \mathrm{P} 2$ and P3 showed strong predominant $\mathrm{LH}$ activation (0.69 and 0.89 , resp.). Regarding successful verb naming, P1 showed a symmetric activation pattern $(-0.21), \mathrm{P} 2$ showed strong predominant $\mathrm{LH}$ activation (0.76), and $\mathrm{P} 3$ showed weak predominant $\mathrm{LH}$ activation (0.36).

3.3. Discussion. The present study aimed to explore maladaptive plasticity, defined as the production of semantic paraphasia, in oral verb naming. Three participants with nonfluent chronic aphasia were examined in the context of a picture-naming task during event-related fMRI scanning. Activation patterns related to the production of semantic paraphasia were obtained and compared to our previous study on adaptive plasticity, that is, successful verb naming [16]. For each participant, the relative contribution of the $\mathrm{RH}$ and $\mathrm{LH}$ to the production of semantic paraphasia and successful verb naming was determined by calculating an LI.

Results show that the production of semantic paraphasia was associated with the significant activation of right and left hemisphere areas in all three participants. All of these areas are reported to sustain normal language processing in healthy adults [46] and particularly verb production [6-11]. The recruitment of these areas may reflect the attempt to find the correct target verb; however, the attempt to compensate for the system's damaged components is not sufficient and leads to semantic paraphasia that is in some way related to the target word. In addition, the production of semantic paraphasia was associated with specific activation patterns in all participants. This may reflect the impact of individual factors such as lesion location and extension, time elapsed after stroke, age, and education level, all of which have been shown to influence language representation and processing [47-51]. Also, specificities in the mechanisms underlying the production of semantic paraphasia between participants may explain these differences. For example, research on cognitive mechanisms underlying the production of semantic paraphasia shows that these may result from impaired phonological processing or impaired semantic processing or a combination of both impairments [5]. In the present study, we did not examine the degree of relative impairment at either of these processing levels in each participant. Therefore, we cannot exclude the possibility that the mechanisms underlying the production of semantic paraphasia may have differed between participants; this may explain why each participant showed specific activation patterns in relation to the production of paraphasia.

The present study also compared the activation patterns related to the production of semantic paraphasia to our previous study on adaptive plasticity, that is, successful verb naming [16]. In each participant, a number of common activation patterns were observed for semantic paraphasia and successful naming. For P1, these included the precentral 


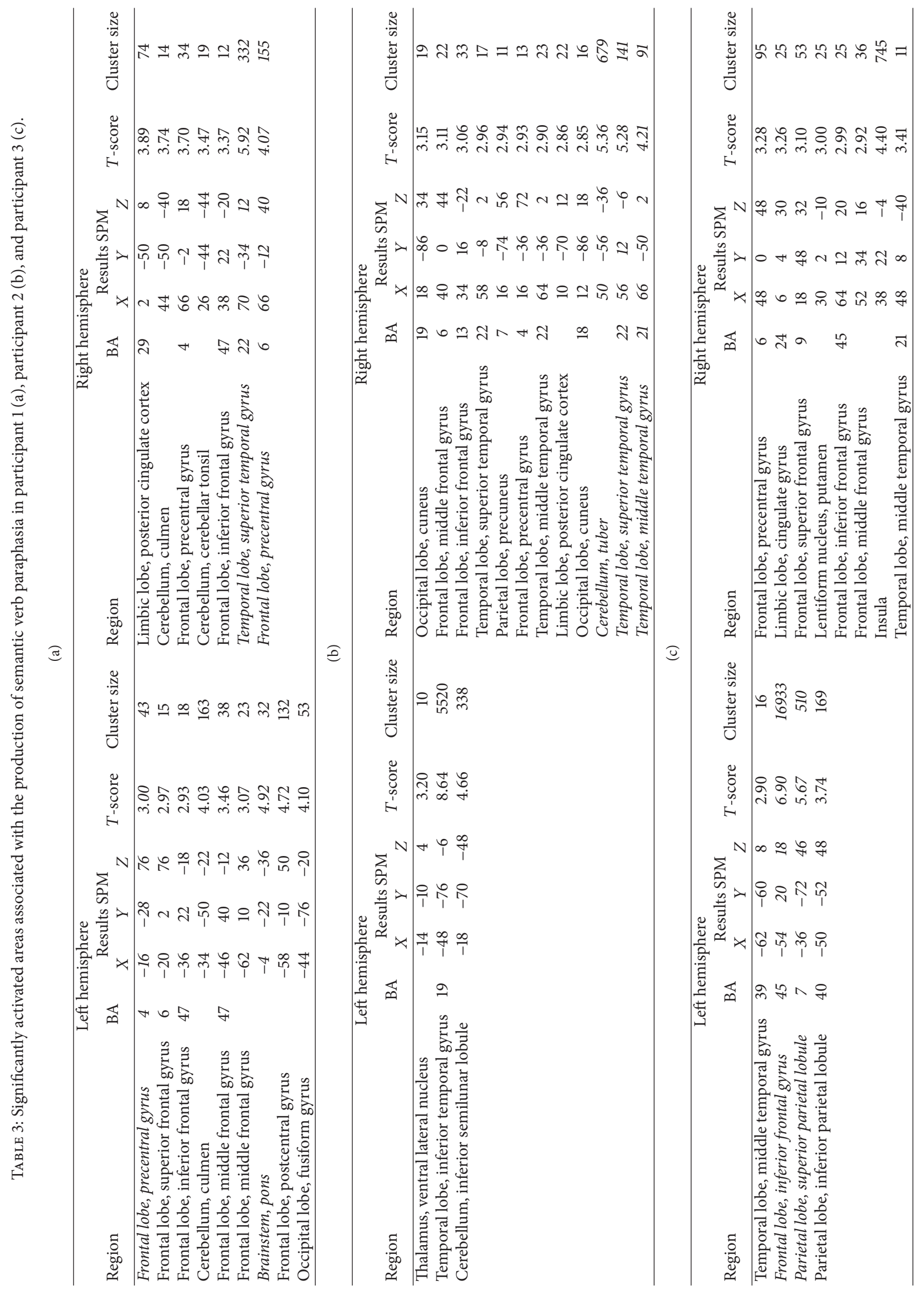




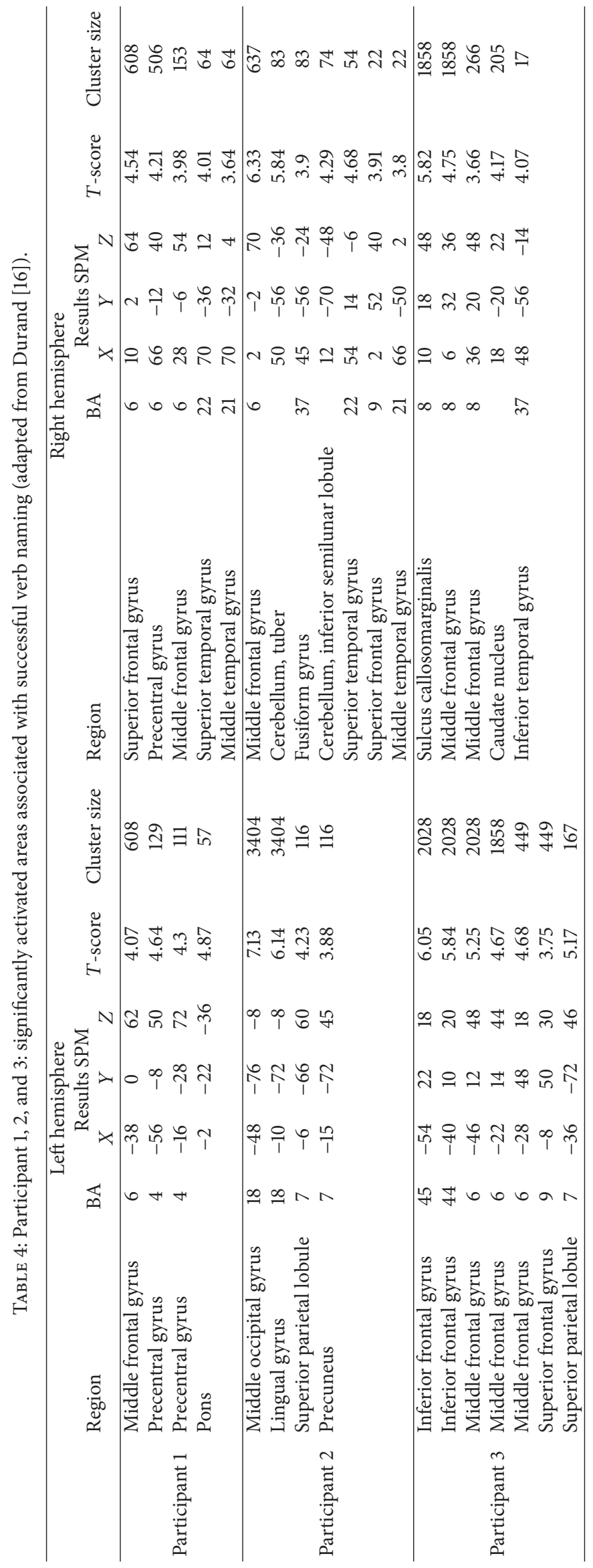


TABLE 5: Lateralization indexes related to maladaptive plasticity, that is production of semantic paraphasia, and adaptive plasticity, that is, successful naming, for each participant. A lateralization index ranging from -0.25 to 0.25 represents a symmetric contribution of the left and right hemispheres to processing (participant 1), whereas a positive value indicates a predominant $\mathrm{RH}$ contribution to processing (participants 2 and 3) [29].

\begin{tabular}{lccc}
\hline & Participant 1 & Participant 2 & Participant 3 \\
\hline $\begin{array}{l}\text { Brain activation map for } \\
\text { semantic paraphasia }\end{array}$ & -0.11 & 0.69 & 0.89 \\
\hline $\begin{array}{l}\text { Brain activation map for } \\
\text { successful naming }\end{array}$ & -0.21 & 0.76 & 0.36 \\
\hline
\end{tabular}

gyrus bilaterally, the left brainstem (pons), and the right STG; for P2, these included the right cerebellum (tuber), the right STG, and the right MTG; and for P3, these included the left IFG and the left superior parietal lobule.

Interestingly, also these areas are known for their contribution to language processing in healthy adults and, particularly, sustaining verb production. Some of these areas are known to be involved in lexicosemantic processing. The precentral gyrus is known for its role in action semantics [911] and the left precentral gyrus is part of a well-known leftlateralized semantic processing circuit [52-54]. The left IFG is involved in lexicosemantic processing [55] and significant activation of the left superior parietal lobule is related to verb production [7]. Further, the right homologue of the left STG is involved in verb production [7]. Besides these areas involved in lexicosemantics, there are common areas for semantic paraphasia and successful naming that are involved in phonological encoding, articulation, and motor speech. The left IFG and the left STG are involved in phonological processing $[56,57]$. The left IFG, left MTG, and cerebellum, together with the primary motor cortex (part of the precentral gyrus), support articulatory planning in speech [22, 5759]. Regarding the left brainstem (pons) and the cerebellum (tuber), they are part of a cerebrocerebellar loop, sustaining articulation and motor speech stages of word production $[60,61]$.

The finding that our three participants showed common significant activation patterns during both semantic paraphasia and successful naming may again reflect an attempt of the system to find the correct target verb; sometimes the attempt is successful, and other times it is not. The production of semantic paraphasia may represent a nonefficient system's attempt to compensate for its damaged components, which leads to the selection of error production that is in some way related to the target word. Conversely, successful naming may reflect a function of the spared tissue or an adaptive compensation for the damaged language components, leading to activation of the correct target word. Moreover, the finding of common significant activation patterns during both semantic paraphasia and successful naming also suggests that segregation of brain areas provides only a partial view of the neural basis of verb anomia and successful verb naming and indicates the need to involve network approaches which better capture the complexity of neuroplasticity mechanisms in anomia recovery.

Concerning the lateralization of processing, the contributions of the $\mathrm{LH}$ and $\mathrm{RH}$ to semantic paraphasia and/or to successful naming are still not totally clear. The LI results of the present study show that both hemispheres contribute to the production of semantic paraphasia and successful naming. RH activation not only is related to the production of semantic paraphasia, but can also be related to successful naming. Therefore, in the present study, $\mathrm{RH}$ activation may correspond to efficient compensation in the context of adaptive plasticity processes. This is in line with studies reporting $\mathrm{RH}$ activation in the context of successful naming in persons with aphasia [18] and also in healthy participants [23].

The finding that the extent of $\mathrm{RH}$ recruitment differed between the three participants might be attributed to lesion size $[12,27]$. Larger lesions (associated with poor recovery of language functions) are associated with RH contribution, while in the case of small LH lesions the left perilesional cortex can sustain language recovery. This mechanism is supported by the present data. P1 presents a large lesion and shows a symmetric activation pattern during both semantic paraphasia and successful naming. In contrast, the $\mathrm{LI}$ of $\mathrm{P} 2$ and $\mathrm{P} 3$ reflects predominant $\mathrm{LH}$ activation in the presence of smaller LH damage and smaller error rates. The observation of a larger number of semantic verb paraphasia types in $\mathrm{P} 1$ can also be related to $\mathrm{RH}$ semantic processing abilities. Therefore, it is possible that the $\mathrm{RH}$ has access to underspecified semantic representations [25] which may favor the production of semantic paraphasia. However, $\mathrm{RH}$ activation in the context of aphasia recovery may reflect the system's attempt to compensate for its damaged components and, to some extent, support access to the correct target word. Therefore, in these three participants, the production of semantic verb paraphasia may reflect an attempt to reach the target in the recovery process.

In summary, these results show that while the global activation pattern differs between the participants, the activation patterns related to maladaptive neuroplasticity and adaptive neuroplasticity comprise a number of common areas. Also, the relative contribution of the left and right hemispheres to maladaptive and adaptive plasticity is not totally clear. This finding challenges the dichotomic distinction between the maladaptive and adaptive roles of the right and left hemispheres, respectively. The present results show that $\mathrm{RH}$ recruitment may be associated with adaptive plasticity mechanisms supporting recovery from anomia. Therefore, these findings raise questions regarding the generalizability of rTMS/tDCS studies reporting the advantages of selectively inhibiting the $\mathrm{RH}$ homologue of Broca's area to trigger anomia recovery [32-35]. The present findings suggest that inhibiting these areas may, at least in some cases, prevent the expression of the adaptive potential of the $\mathrm{RH}$ to support anomia recovery and/or abort the emergence of semantic strategies that may contribute to attenuating the effects of anomia in everyday communication.

The present results support a less dichotomic perspective with regard to the contribution of the right and left hemispheres to recovery from anomia and indicate the 
importance of adopting a wider perspective when examining the neural basis of anomia recovery. In particular, functional connectivity approaches offer an interesting alternative to the segregation perspective, as they allow considering the dynamic changes that occur within a specific brain network, which may be composed of a similar set of areas. The functional connectivity approach highlights changes in network configuration and activity, depending on a variety of factors, such as complexity level and type of task. Future functional connectivity studies on the neural basis of anomia recovery may help unravel the complex mechanisms underlying neuroplasticity in anomia recovery.

A limitation of the present study is the small number of participants and the fact that all of them were males. However, single-case studies provide important information regarding the variety of idiosyncratic activation patterns in paraphasia and successful naming. Nevertheless, larger samples, including males and females, need to be examined to further elucidate the role of right hemisphere areas and circuits in the adaptive or maladaptive mechanisms that sustain anomia recovery.

\section{Conclusion}

The present study explored maladaptive plasticity in persistent verb anomia by analyzing activation patterns associated with semantic verb paraphasia production in three male participants with chronic nonfluent aphasia. The results show that activation patterns associated with paraphasia production differ across the three participants. This reflects individual factors such as lesion location, time after onset, and the nature of the underlying processing deficits in the context of anomia. The present study also compared the activation patterns related to the production of semantic paraphasia to our previous study on adaptive plasticity, that is, successful verb naming [16]. Interestingly, our three participants showed common significant activation patterns during both semantic paraphasia and successful naming. Finally, the data show that both the $\mathrm{LH}$ and the $\mathrm{RH}$ are related to the production of semantic paraphasia, thereby questioning the idea of a maladaptive role of the RH. Our findings have implications for future studies aiming at inhibiting or activating specific areas in the context of rTMS/tDCS and suggest that the neural basis of paraphasia and successful naming is not mutually exclusive but may reflect dynamic processes within a relatively limited set of contributing areas.

\section{Competing Interests}

The authors declare that there is no conflict of interests regarding the publication of this paper.

\section{Acknowledgments}

This study was supported by the Trans-Atlantic Neuroscience Teaching Network (TANTEN).

\section{References}

[1] I. Papathanasiou and P. Coppens, Aphasia and Related Neurogenic Communication Disorders: Basis Concepts and Operational Definitions, Jones \& Bartlett learning, Burlington, Mass, USA, 2013.

[2] M. Laine and N. Martin, Anomia: Theoretical and Clinical Aspects, Psychology Press, New York, NY, USA, 2006.

[3] J. Druks, "Verbs and nouns-a review of the literature," Journal of Neurolinguistics, vol. 15, no. 3-5, pp. 289-315, 2002.

[4] H. Goodglass and A. Wingfield, Word-Finding Deficits in Aphasia: Brain-Behaviour Relations and Clinical Symptomatology, Academic Press, San Diego, Calif, USA, 1997.

[5] A. Caramazza and A. E. Hillis, "Where do semantic errors come from?” Cortex, vol. 26, no. 1, pp. 95-122, 1990.

[6] K. A. Shapiro, A. Pascual-Leone, F. M. Mottaghy, M. Gangitano, and A. Caramazza, "Grammatical distinctions in the left frontal cortex," Journal of Cognitive Neuroscience, vol. 13, no. 6, pp. 713720, 2001.

[7] K. A. Shapiro, L. R. Moo, and A. Caramazza, "Cortical signatures of noun and verb production," Proceedings of the National Academy of Sciences of the United States of America, vol. 103, no. 5, pp. 1644-1649, 2006.

[8] K. A. Shapiro, F. M. Mottaghy, N. O. Schiller et al., "Dissociating neural correlates for nouns and verbs," NeuroImage, vol. 24, no. 4, pp. 1058-1067, 2005.

[9] C. A. Porro, M. P. Francescato, V. Cettolo et al., "Primary motor and sensory cortex activation during motor performance and motor imagery: a functional magnetic resonance imaging study," The Journal of Neuroscience, vol. 16, no. 23, pp. 76887698, 1996

[10] F. Pulvermüller, "Brain mechanisms linking language and action," Nature Reviews Neuroscience, vol. 6, no. 7, pp. 576-582, 2005.

[11] F. Pulvermüller, O. Hauk, V. V. Nikulin, and R. J. Ilmoniemi, "Functional links between motor and language systems," European Journal of Neuroscience, vol. 21, no. 3, pp. 793-797, 2005.

[12] J. Fridriksson, J. M. Baker, and D. Moser, "Cortical mapping of naming errors in aphasia," Human Brain Mapping, vol. 30, no. 8, pp. 2487-2498, 2009.

[13] S. F. Cappa, "Recovery from aphasia: why and how?" Brain and Language, vol. 71, no. 1, pp. 39-41, 2000.

[14] J. Grafman, "Conceptualizing functional neuroplasticity," Journal of Communication Disorders, vol. 33, no. 4, pp. 345-356, 2000.

[15] J. A. Kleim and T. A. Jones, "Principles of experience-dependent neural plasticity: implications for rehabilitation after brain damage," Journal of Speech, Language, and Hearing Research, vol. 51, no. 1, pp. S225-S239, 2008.

[16] E. Durand, Récupération de la capacité à dénommer des actions dans l'aphasie chronique: étude des effets d'une thérapie sémantique auprès de trois participants [M.S. thesis], École d'Orthophonie et d'Audiologie, Faculté de Médecine, Université de Montréal, Montreal, Canada, 2011.

[17] L. J. Harris, "Broca on cerebral control for speech in righthanders and left-handers: a note on translation and some further comments," Brain and Language, vol. 45, no. 1, pp. 108120, 1993

[18] C. Anglade, A. Thiel, and A. I. Ansaldo, "The complementary role of the cerebral hemispheres in recovery from aphasia after stroke: a critical review of literature," Brain Injury, vol. 28, no. 2, pp. 138-145, 2014. 
[19] C. Code, Language Aphasia and the Right Hemisphere, John Wiley \& Sons, England, UK, 1987.

[20] W.-D. Heiss, J. Kessler, A. Thiel, M. Ghaemi, and H. Karbe, "Differential capacity of left and right hemispheric areas for compensation of poststroke aphasia," Annals of Neurology, vol. 45, no. 4, pp. 430-438, 1999.

[21] D. Saur, R. Lange, A. Baumgaertner et al., "Dynamics of language reorganization after stroke," Brain, vol. 129, no. 6, pp. 1371-1384, 2006.

[22] I. K. Christoffels, E. Formisano, and N. O. Schiller, "Neural correlates of verbal feedback processing: an fMRI study employing overt speech," Human Brain Mapping, vol. 28, no. 9, pp. 868879, 2007.

[23] G. Raboyeau, X. De Boissezon, N. Marie et al., "Right hemisphere activation in recovery from aphasia: lesion effect or function recruitment?" Neurology, vol. 70, no. 4, pp. 290-298, 2008.

[24] S. C. Blank, H. Bird, F. Turkheimer, and R. J. S. Wise, "Speech production after stroke: the role of the right pars opercularis," Annals of Neurology, vol. 54, no. 3, pp. 310-320, 2003.

[25] M. Jung-Beeman, "Bilateral brain processes for comprehending natural language," Trends in Cognitive Sciences, vol. 9, no. 11, pp. 512-518, 2005.

[26] L. Bonilha, E. Gleichgerrcht, T. Nesland, C. Rorden, and J. Fridriksson, "Success of anomia treatment in aphasia is associated with preserved architecture of global and left temporal lobe structural networks," Neurorehabilitation and Neural Repair, vol. 30, no. 3, pp. 266-279, 2016.

[27] W.-D. Heiss, H. Karbe, G. Weber-Luxenburger et al., "Speechinduced cerebral metabolic activation reflects recovery from aphasia," Journal of the Neurological Sciences, vol. 145, no. 2, pp. 213-217, 1997.

[28] A. Thiel, B. Habedank, K. Herholz et al., "From the left to the right: how the brain compensates progressive loss of language function," Brain and Language, vol. 98, no. 1, pp. 57-65, 2006.

[29] S. Lehéricy, L. Cohen, B. Bazin et al., "Functional MR evaluation of temporal and frontal language dominance compared with the Wada test," Neurology, vol. 54, no. 8, pp. 1625-1633, 2000.

[30] K. Marcotte and A. I. Ansaldo, "The neural correlates of semantic feature analysis in chronic aphasia: discordant patterns according to the etiology," Seminars in Speech and Language, vol. 31, no. 1, pp. 52-63, 2010.

[31] P. Vitali, J. Abutalebi, M. Tettamanti et al., "Training-induced brain remapping in chronic aphasia: a pilot study," Neurorehabilitation and Neural Repair, vol. 21, no. 2, pp. 152-160, 2007.

[32] P. I. Martin, M. A. Naeser, H. Theoret et al., "Transcranial magnetic stimulation as a complementary treatment for aphasia," Seminars in Speech and Language, vol. 25, no. 2, pp. 181-191, 2004.

[33] M. A. Naeser, P. I. Martin, M. Nicholas et al., "Improved picture naming in chronic aphasia after TMS to part of right Broca's area: an open-protocol study," Brain and Language, vol. 93, no. 1, pp. 95-105, 2005.

[34] J. Fridriksson, J. D. Richardson, J. M. Baker, and C. Rorden, "Transcranial direct current stimulation improves naming reaction time in fluent aphasia: a double-blind, sham-controlled study," Stroke, vol. 42, no. 3, pp. 819-821, 2011.

[35] A. Monti, R. Ferrucci, M. Fumagalli et al., "Transcranial direct current stimulation (tDCS) and language," Journal of Neurology, Neurosurgery and Psychiatry, vol. 84, no. 8, pp. 832-842, 2013.
[36] G. Tononi, G. M. Edelman, and O. Sporns, "Complexity and coherency: integrating information in the brain," Trends in Cognitive Sciences, vol. 2, no. 12, pp. 474-484, 1998.

[37] L. Cloutman, R. Gottesman, P. Chaudhry et al., "Where (in the brain) do semantic errors come from?" Cortex, vol. 45, no. 5, pp. 641-649, 2009.

[38] K. Marcotte, V. Perlbarg, G. Marrelec, H. Benali, and A. I. Ansaldo, "Default-mode network functional connectivity in aphasia: therapy-induced neuroplasticity," Brain and Language, vol. 124, no. 1, pp. 45-55, 2013.

[39] L. Ghazi Saidi, V. Perlbarg, G. Marrelec, M. Pélégrini-Issac, H. Benali, and A.-I. Ansaldo, "Functional connectivity changes in second language vocabulary learning," Brain and Language, vol. 124, no. 1, pp. 56-65, 2013.

[40] K. Marcotte, D. Adrover-Roig, B. Damien et al., "Therapyinduced neuroplasticity in chronic aphasia," Neuropsychologia, vol. 50, no. 8, pp. 1776-1786, 2012.

[41] J.-L. Nespoulous, A. Lecours, D. Lafond, A. Lemay, M. Puel, and Y. Joanette, Protocole Montréal-Toulouse d'examen linguistique de laphasie MT 86. Module standard initial: MIB, Laboratoire Théophile-Alajouanine, Montréal, Canada, 1986.

[42] J. G. Snodgrass and M. Vanderwart, "A standardized set of 260 pictures: norms for name agreement, image agreement, familiarity, and visual complexity," Journal of Experimental Psychology: Human Learning and Memory, vol. 6, no. 2, pp. 174215, 1980

[43] S. P. Limited, ColorCards, Speechmark, England, UK.

[44] J. L. Lancaster, M. G. Woldorff, L. M. Parsons et al., "Automated talairach atlas labels for functional brain mapping," Human Brain Mapping, vol. 10, no. 3, pp. 120-131, 2000.

[45] A. C. Evans, D. L. Collins, S. R. Mills, E. D. Brown, R. L. Kelly, and T. M. Peters, "3D statistical neuroanatomical models from 305 MRI volumes," in Proceedings of the IEEE Nuclear Science Symposium \& Medical Imaging Conference, pp. 18131817, November 1993.

[46] C. J. Price, "The anatomy of language: a review of $100 \mathrm{fMRI}$ studies published in 2009," Annals of the New York Academy of Sciences, vol. 1191, pp. 62-88, 2010.

[47] H. Duffau, S. Moritz-Gasser, and E. Mandonnet, "A reexamination of neural basis of language processing: proposal of a dynamic hodotopical model from data provided by brain stimulation mapping during picture naming," Brain and Language, vol. 131, pp. 1-10, 2014.

[48] P. J. Eslinger and A. R. Damasio, "Age and type of aphasia in patients with stroke," Journal of Neurology Neurosurgery and Psychiatry, vol. 44, no. 5, pp. 377-381, 1981.

[49] S. Jarso, M. Li, A. Faria et al., "Distinct mechanisms and timing of language recovery after stroke," Cognitive Neuropsychology, vol. 30, no. 7-8, pp. 454-475, 2013.

[50] P. M. Pedersen, K. Vinter, and T. S. Olsen, "Aphasia after stroke: type, severity and prognosis: the Copenhagen aphasia study," Cerebrovascular Diseases, vol. 17, no. 1, pp. 35-43, 2004.

[51] A. C. Laska, A. Hellblom, V. Murray, T. Kahan, and M. Von Arbin, "Aphasia in acute stroke and relation to outcome," Journal of Internal Medicine, vol. 249, no. 5, pp. 413-422, 2001.

[52] T. R. Barrick, I. N. Lawes, C. E. Mackay, and C. A. Clark, "White matter pathway asymmetry underlies functional lateralization," Cerebral Cortex, vol. 17, no. 3, pp. 591-598, 2007.

[53] M. Catani, D. K. Jones, and D. H. Ffytche, "Perisylvian language networks of the human brain," Annals of Neurology, vol. 57, no. 1, pp. 8-16, 2005. 
[54] E. Durand and A. I. Ansaldo, "Recovery from anomia following semantic feature analysis: therapy-induced neuroplasticity relies upon a circuit involving motor and language processing areas," Mental Lexicon, vol. 8, no. 2, pp. 195-215, 2013.

[55] J.-F. Démonet, F. Chollet, S. Ramsay et al., "The anatomy of phonological and semantic processing in normal subjects," Brain, vol. 115, no. 6, pp. 1753-1768, 1992.

[56] S. Bookheimer, "Functional MRI of language: new approaches to understanding the cortical organization of semantic processing," Annual Review of Neuroscience, vol. 25, pp. 151-188, 2002.

[57] R. Mayeux and E. Kandel, Natural Language, Disorders of Language, and Other Localizable Disorders of Cognitive Function, Elsevier, New York, NY, USA, 1985.

[58] F. H. Guenther, "Cortical interactions underlying the production of speech sounds," Journal of Communication Disorders, vol. 39, no. 5, pp. 350-365, 2006.

[59] P. Indefrey and W. Levelt, The Neural Correlates of Language Production, MIT Press, Cambridge, Mass, USA, 2000.

[60] J. D. Schmahmann and D. N. Pandyaf, "The cerebrocerebellar system," International Review of Neurobiology, vol. 41, pp. 31-60, 1997.

[61] E. V. Sullivan, "Compromised pontocerebellar and cerebellothalamocortical systems: speculations on their contributions to cognitive and motor impairment in nonamnesic alcoholism," Alcoholism: Clinical and Experimental Research, vol. 27, no. 9, pp. 1409-1419, 2003. 

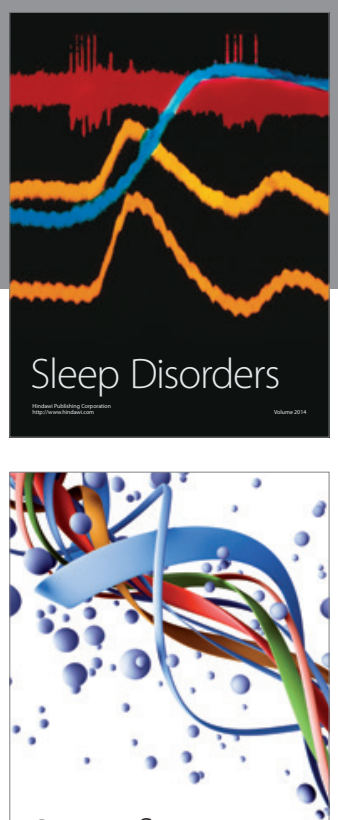

Scientifica
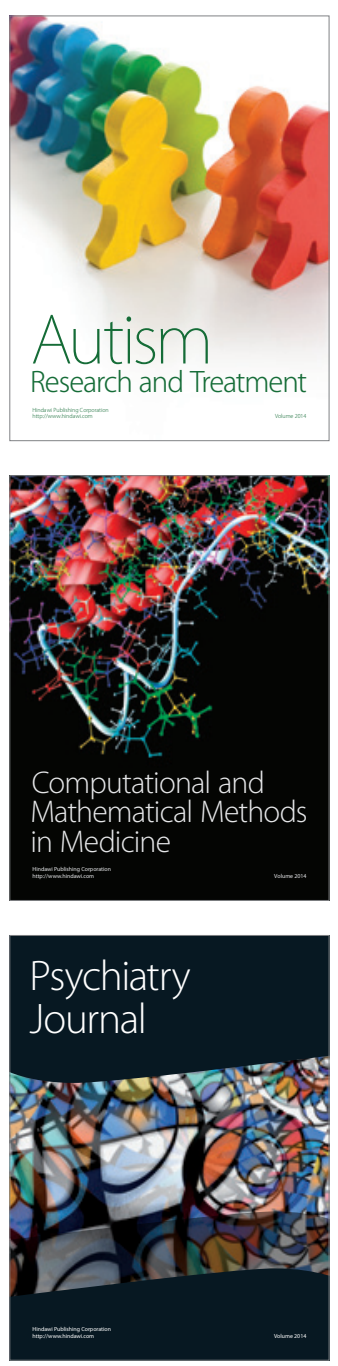
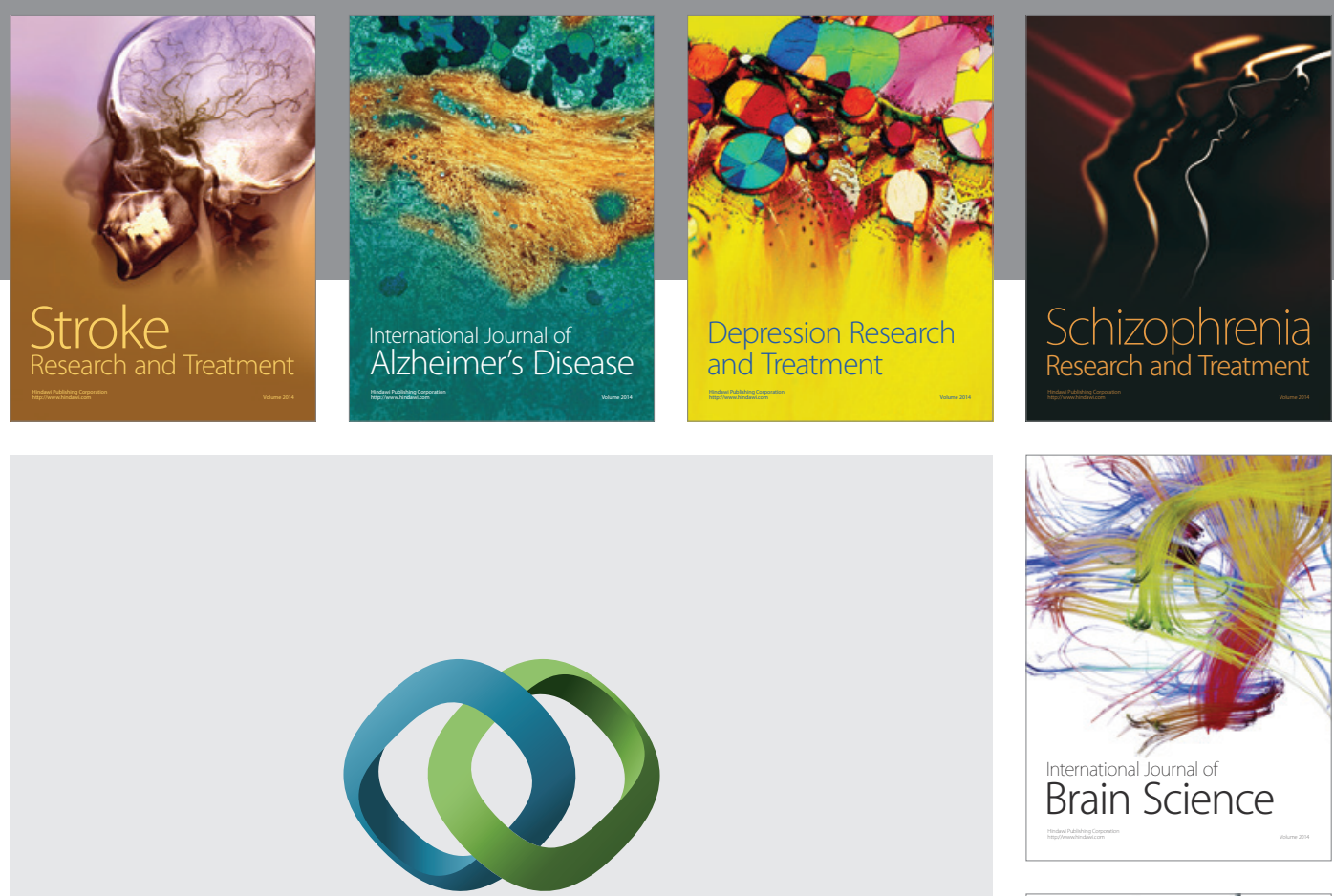

\section{Hindawi}

Submit your manuscripts at

http://www.hindawi.com
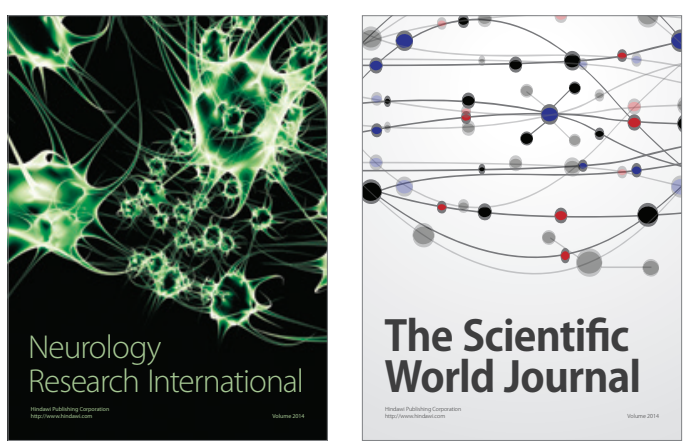

The Scientific World Journal

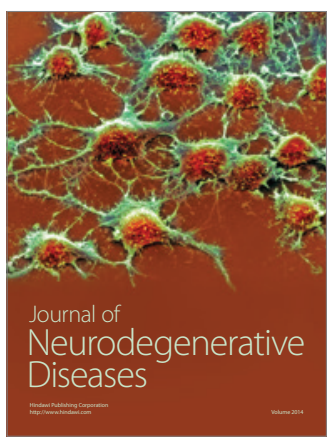

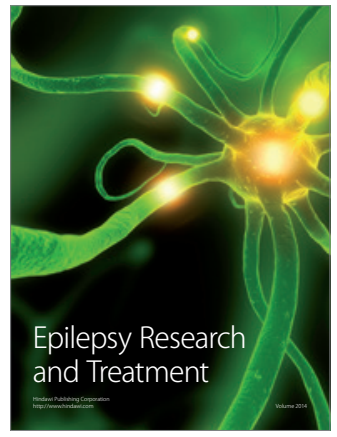

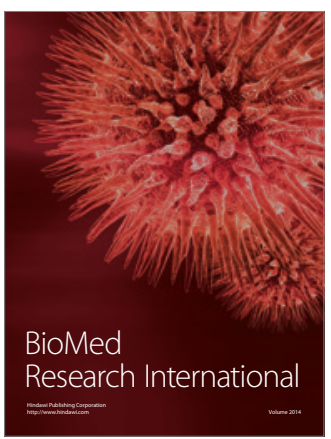

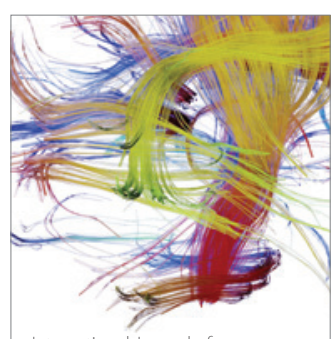

Brain Science

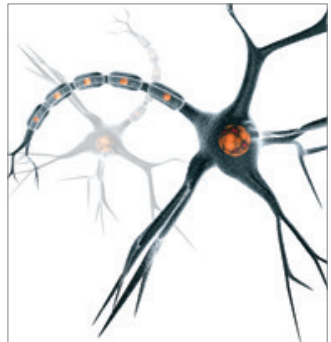

Neural Plasticity
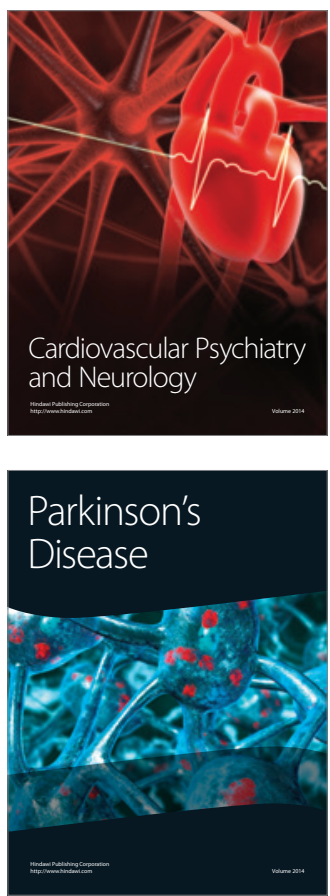